\title{
Kcna1 Gene Deletion Lowers the Behavioral Sensitivity of Mice to Small Changes in Sound Location and Increases Asynchronous Brainstem Auditory Evoked Potentials But Does Not Affect Hearing Thresholds
}

\author{
Paul D. Allen ${ }^{1}$ and James R. Ison ${ }^{1,2}$ \\ ${ }^{1}$ Department of Neurobiology and Anatomy, University of Rochester Medical Center, Rochester, New York 14642 and ${ }^{2}$ Department of Brain and Cognitive \\ Sciences, University of Rochester, Rochester, New York 14627
}

\begin{abstract}
Sound localization along the azimuth depends on the sensitivity of binaural nuclei in the auditory brainstem to small differences in interaural level and timing occurring within a submillisecond epoch and on monaural pathways that transmit level and timing cues with high temporal fidelity to insure their coincident arrival at the binaural targets. The soma and axons of these brainstem neurons are heavily invested with ion channels containing the low-threshold potassium channel subunit Kv1.1, which previous in vitro and in vivo studies suggest are important for regulating their high input- output correspondence and temporal synchrony. We compared awake Kcna1-null mutant $(\mathrm{Kcna1}-/-)$ mice lacking Kv1.1 with Kcna1 +/+ mice to determine whether Kv1.1 activity contributes to sound localization and examined anesthetized mice for absolute hearing thresholds for suprathreshold differences that may be revealed in the waveforms of auditory brainstem response potentials. The awake $-/-$ mice tested with reflex modification audiometry had reduced sensitivity to an abrupt change in the location of a broad band noise compared to $+/+$ mice, while anesthetized $-/-$ mice had normal absolute thresholds for tone pips but a high level of stimulus-evoked but asynchronous background activity. Evoked potential waveforms had progressively earlier peaks and troughs in $-/-$ mice, but the amplitude excursions between adjacent features were identical in the two groups. Their greater excitability and asynchrony in suprathreshold evoked potentials coupled with their normal thresholds suggests that a disruption in central neural processing in $-/-$ mice and not peripheral hearing loss is responsible for their poor sound localization.
\end{abstract}

\section{Introduction}

The ability to locate sounds contributes to spatial orientation and navigation, and also helps humans and other animals segregate overlapping auditory signals, including vocalizations, by their perceived locations (Cherry, 1953; Feng and Ratnam, 2000). The major cues for sound localization are interaural time (ITDs) and intensity differences (IIDs) produced by small variations in the distance between a source and each ear and by sound shadowing of the longer path around the head. Typical detection thresholds in mammals are $<10^{\circ}$ of arc and just $1^{\circ}$ for humans (Hershkowitz and Durlach, 1969), indicating their utilization of ITDs of $10 \mu \mathrm{s}$

Received April 19, 2011; revised Dec. 29, 2011; accepted Jan. 6, 2012.

Author contributions: P.D.A. and J.R.I. designed research; P.D.A. and J.R.I. performed research; P.D.A. and J.R.I. analyzed data; P.D.A. and J.R.I. wrote the paper.

This work was supported by United States Public Health Service NIH-NIA P01 Grant AG09524, NIH-NIDCD P30 Grant DC005409, and the Schmitt Foundation for Integrative Brain Research. We thank Drs. Bruce Tempel, Cornelia Kopp-Scheinpflug, and Gary Paige for discussion and comments on previous versions of this manuscript; Dr. Robert Frisina, Xiaoxia Zhu and Martha Zettel for genotyping the mice; Dr. William O'Neill, Dr. Joseph Walton, and John Housel for their contributions to ABR testing and interpretation; and two anonymous reviewers for their wellinformed comments.

The authors declare no competing financial interests.

Correspondence should be addressed to Dr. Paul D. Allen, Department of Neurobiology and Anatomy, University of Rochester Medical Center, 601 Elmwood Avenue, Box 603 Rochester, NY 14642. E-mail: paul_allen@urmc.rochester.edu.

DOI:10.1523/JNEUROSCI.1958-11.2012

Copyright $\odot 2012$ the authors $\quad 0270-6474 / 12 / 322538-06 \$ 15.00 / 0$ and IIDs of $1 \mathrm{~dB}$ (Mills, 1958). High temporal fidelity of neurotransmission is required for both binaural comparisons, because these IID- and ITD-coding nuclei must integrate only near-coincident neural impulses arriving from each ear (Oertel, 1999; Joris and Yin, 1995). Anatomical and physiological specializations in the brainstem ensure that monaural neurons rapidly transmit their separate inputs in synchrony to their upstream targets and that binaural neurons have brief integration windows (Trussell 1999; Yin, 2002). One significant molecular adaptation in these neurons and in other brainstem nuclei involved in coincidence detection (e.g., octopus cells) (Oertel et al., 2000) is their expression of voltage-sensitive Kv1 channels (Grigg et al., 2000; Rosenberger et al., 2003). The most sensitive subunit in these channels is Kv1.1, which is the focus of the present paper.

In vitro slice studies of a major monaural nucleus in the IID pathway, the medial nucleus of the trapezoid body (MNTB), show that cells from $\mathrm{Kcna1}-/$ - mice lacking Kv1.1 respond with many poorly timed responses to current injection (high "jitter"), while Kcna1 +/+ cells have single onset spikes with stable latencies (Brew et al., 2003; Gittelman and Tempel, 2006). Comparable in vivo studies in anesthetized $-/-$ mice report not hyperexcitability but hypoexcitability to tone pips, though -/cells in the cochlear nucleus $(\mathrm{CN})$ and MNTB again show greater jitter (Kopp-Scheinpflug et al., 2003). More jitter was found also 
in $-/-$ cells of the binaural lateral superior olivary nucleus (LSO), and their sensitivity was restricted to positive IIDs (Karcz et al., 2011). Related work (Karcz, 2011) found that LSO targets in the $-/-$ inferior colliculus (IC) had normal jitter and sensitivity to both positive and negative IIDs, but greater sensitivity to IIDs outside the normal temporal integration window. The in vivo studies also reported at least trends for higher response thresholds in $-/-$ cells (significant in LSO and IC), this not being evident in in vitro studies.

These in vivo reports suggest a direct contribution of Kv1.1 to the neural processing of auditory space, though an alternative hypothesis is that Kcnal deletion simply causes peripheral hearing loss. Here we report that awake $-/-$ mice were indeed less sensitive to changes in sound location in a behavioral task, while anesthetized $-/-$ mice had normal auditory brainstem response (ABR) thresholds, but greater levels of background asynchrony. These data support the conclusion that a disruption in neural processing and not peripheral hearing loss is responsible for their poor sound localization.

\section{Materials and Methods}

Animals. Mice were of the $\mathrm{C} 3 \mathrm{HeB} / \mathrm{FeJ}-\mathrm{Kcna1}^{\mathrm{tm} 1 \mathrm{Tem}}$ strain (Smart et al., 1998), bred in the University of Rochester vivarium from heterozygotic $(+/-)$ breeding pairs obtained from Jackson Laboratory. Genotyping was performed on DNA isolated from tail clips of each mouse, following standard procedures described in protocols available online (http://depts.washington.edu/tempelab/Protocols/KCNA1.html). A total of 63 mice of either sex were tested: 23 in the behavioral experiment $(+/+, 6$ male, 7 female; $-/-, 7$ male, 3 female) and 48 in the ABR experiment $(+/+, 14$ male, 9 female, 1 unknown; $-/-, 12$ male, 10 female, 2 unknown), with 8 mice tested in both experiments. The ABR experiment was administered when the mice were on average $21 \mathrm{~d}$ of age; the behavioral tests began, on average, at $30 \mathrm{~d}$ of age. The behavioral experiment was performed over a period of 7 months, and the mice were selected from the available $+/+$ and $-/-$ mice in that period of time. The ABR data were collected over a period of 15 months, and to minimize any possible litter effects, the groups here were specially selected and comprised of all of the matched $+/+$ and $-/-$ littermates from the larger colony. The University of Rochester Committee on Animal Resources approved all procedures, which were in accord with United States Public Health Service regulations and the Federal Animal Welfare Act.

Auditory brainstem response. Mice were anesthetized with ketamine/ xylazine (120 and $10 \mathrm{mg} / \mathrm{kg}$, respectively) and then placed on a $10-\mathrm{cm}-$ high platform inside a small ( $57 \mathrm{~cm}$ long by $41 \mathrm{~cm}$ wide by $36 \mathrm{~cm}$ high), electrically shielded, soundproofed booth (IAC) lined with $4.5-\mathrm{cm}$-thick echo-attenuating acoustic foam (Sonex; Illbruck Acoustic). Each mouse was arranged in a prone position facing a broad-band electrostatic loudspeaker system [Tucker-Davis Technology (TDT) ES1 with an ED1 speaker driver] located $10 \mathrm{~cm}$ from the head, and was kept warm during the procedure with a circulating-water heating pad. Miniature subdermal needle electrodes (Nicolet) were inserted at the vertex (reference electrode), over the bulla (active electrode), and just above the hindlimb (ground electrode). Calibrated tone bursts ( $5 \mathrm{~ms}$ duration, $0.5 \mathrm{~ms}$ risefall time, phase alternating $90^{\circ}$ ) were synthesized with SigGenRP software on a TDT RP2.1 Real-Time Processor and presented at a rate of 10 per second at frequencies of $3,6,12,16,24,32$, and $48 \mathrm{kHz}$. The software included an artifact detector that rejected outlier responses. Average ABR waveforms (150 repetitions by two duplicates) were recorded at each tested frequency/amplitude combination and filtered from 3 to $1000 \mathrm{~Hz}$. Each $10 \mathrm{~ms}$ waveform was comprised of 500 points $(0.02 \mathrm{~ms}$ sample period). Every waveform was normalized with a DC correction so that the sum of the 500 points was $0 \mu \mathrm{V}$. At each frequency, the amplitude of the signal was automatically attenuated (TDT PA5) in $5 \mathrm{~dB}$ steps between samples from $90 \mathrm{~dB}$ SPL (but $80 \mathrm{~dB}$ SPL maximum at $48 \mathrm{kHz}$ ) until the wave IV-V complex of the ABR waveform was no longer visually distinguishable from background in duplicate traces in the judgment of a highly experienced technician. By convention, threshold was established as the response at $5 \mathrm{~dB}$ above this final level. Suprathreshold amplitude measures were later analyzed from these waveforms and are reported here for the $16 \mathrm{kHz}$ test frequency presented at $80 \mathrm{~dB}$ SPL.

Behavioral measure of auditory spatial acuity. The auditory spatial acuity of mice was determined using reflex modification audiometry as described previously (Allen and Ison, 2010). Briefly, a broadband noise was switched abruptly between speakers that were separated by $7.5,22.5,45$, 90 , and $180^{\circ}$ in the frontal plane of the mouse [this switch being the prepulse (PP)], with the order of the five angular separations across test days counterbalanced across mice. The mouse's detection of the PP was demonstrated in the inhibition (PPI) of the acoustic startle response (ASR) to a noise burst probe [the reflex-eliciting stimulus (ES)] when the probe was presented shortly after the change in the location of the background noise (a prepulse trial) compared to the response amplitude when it was presented without any prior change in the background noise (a control trial). A schematic diagram of the stimulus arrangement is provided in Figure 1.

The experiment was conducted within a large ( $2.7 \mathrm{~m}$ long, $1.8 \mathrm{~m}$ wide, $2.4 \mathrm{~m}$ high) sound-attenuating IAC room with echo-attenuating Sonex acoustical foam lining the walls. The ES was generated using a TDT RP2.1 Real-time Processor amplified with an Adcom GFA-535 II amplifier and broadcast via a Yamaha JA4281B compression tweeter. This stimulus was $110 \mathrm{~dB}$ SPL, but at just $20 \mathrm{~ms}$ duration its subjective level for a human listener approximated that of a hand clap at a distance of $30 \mathrm{~cm}$. The background noise stimulus at $70 \mathrm{~dB}$ SPL was digitally generated using a second RP2.1 processor and broadcast from one of two matched TDT ES1 electrostatic speakers located $50 \mathrm{~cm}$ from the mouse's head in the azimuthal plane. The force of the ASR was transduced by an accelerometer, and its amplitude was scored as the integrated root mean square (RMS) voltage of this signal for $100 \mathrm{~ms}$ after the delivery of the ES. The experimental stimuli were controlled and the responses recorded by a PC using a custom LabView (National Instruments) front-end. On each test day, there were 11 presentations of each PP condition and 22 control startle responses. Prepulse stimuli were presented at 13 different interstimulus interval (ISI) conditions (1, 2, 5, 10, 20, 30, 40, 50, 60, 100, 150, 200 , or $300 \mathrm{~ms}$ ) before the startle stimulus to determine the peak time of their effect. The intertrial interval, during which the background noise was continuously present, averaged $20 \mathrm{~s}$ and was randomly selected from the range 15 to $25 \mathrm{~s}$. Each testing session lasted $\sim 60 \mathrm{~min}$, with one session for each angular separation, each separated by at least one rest day. Scheduling constraints resulted in not all animals receiving all five stimulus conditions.

Data analysis. Prepulse inhibition scores were calculated as the ratio of the each subject's mean startle response amplitude in the prepulse condition $\left(\mathrm{ASR}_{\mathrm{p}}\right)$ compared with the no-prepulse control baseline $\left(\mathrm{ASR}_{\mathrm{c}}\right)$, $\mathrm{PPI}=1-\left[\mathrm{ASR}_{\mathrm{p}} / \mathrm{ASR}_{\mathrm{c}}\right]$. Repeated-measures ANOVAs with ISI duration as the within-subject variable were performed with SPSS version 18 (IBM-SPSS), and genotype as well as angular separation were taken as between-subjects variables; in fact, angular separation was a withinsubject variable, but because some animals did not complete all tests, there would have been many missing values for a within-subject analysis of angular separation. For this reason it was treated as a between-subjects variable, which provides a conservative estimate of its statistical significance. The $p$ values provided by the ANOVA were adjusted via the Hunyh-Feldt method for nonhomogeneity of between-cell correlations. Graphical presentation of the data and supplemental $t$ tests on specific conditions within and between subjects used GraphPad Prism (version 4.2).

ABR waveforms for the $16 \mathrm{kHz}, 80 \mathrm{~dB}$ SPL stimulus condition were adjusted to equalize the mean amplitude of the first $0.5 \mathrm{~ms}$ portion of each waveform and the zero time base was adjusted to align the latency of the first ABR peak (P1) at $0 \mathrm{~ms}$. The morphology of the waveform was first analyzed with a series of mixed-design ANOVAs in 2 ms blocks beginning $2 \mathrm{~ms}$ before $\mathrm{P} 1$, with time point as the within-subject variable (99 levels) and genotype as the between-subjects variable. Given the significant interactions between genotype and time points in these analyses, a more detailed time analysis began with the mean difference between the $+/+$ and the $-/-$ waveforms and then examining the regions that exceeded the $95 \%$ confidence level for these differences. 




Figure 1. Schematic description of the behavioral experiment. Two speakers were located $1 \mathrm{~m}$ from the mouse and positioned in the azimuthal plane symmetrically around the midline, with angular separations from 7 to $180^{\circ}$. The speaker presenting the startle stimulus was located $10 \mathrm{~cm}$ directly above the mouse's head. Each trial began with the broad-band noise presented from Speaker 1 on the right, playing at $70 \mathrm{~dB}$ SPL for $\sim 20$ s. The noise burst that elicited the startle response was either presented with no prior stimulus change (a control trial) or, on a prepulse trial, the noise was shifted to Speaker 2 on the left, and the startle stimulus was presented after an interstimulus delay interval varying between 1 and 300 ms. Two seconds later, the noise reset to Speaker 1 on the right, initiating the next trial. The startle response was determined by integrating the activity of the mouse in the $100 \mathrm{~ms}$ window following the onset of the startle stimulus.

\section{Results}

Normal thresholds but more asynchronous auditory brainstem responses and reduced latencies in mice lacking Kv1.1

Auditory brainstem response thresholds were recorded as a measure of absolute threshold sensitivity for tone pip stimuli in the $+/+$ and $-/-$ mice and also to examine possible differences in brainstem neural excitability and the timing of their suprathreshold ABR waveforms. Figure 2 describes these results. The threshold data shown in Figure $2 \mathrm{~A}$ are typical for mice, with their best hearing at $16 \mathrm{kHz}$ and thresholds rising steeply at lower frequencies, and more gradually for higher frequencies. The threshold differences between the two groups of mice were small: the ANOVA of these data provided a significant effect for test frequency $\left(F_{(6,276)}=518.61 ; p<0.001 ; \eta_{p}^{2}=0.92\right)$, but the effect of the Kcnal genotype was not significant $(p>0.10)$. The $95 \%$ confidence interval for the mean threshold difference across test frequencies ranged from $4.0 \mathrm{~dB}$, favoring the $+/+$ mice, to 0.42 $\mathrm{dB}$, favoring the $-/-$ mice.

Figure $2 B$ shows the average waveforms in the two groups for $16 \mathrm{kHz}$ at $80 \mathrm{~dB}$ after each individual waveform was time and amplitude normalized as described above. Overall the mean ABR amplitude for the $-/-$ mice was greater than that of the $+/+$ mice $\left(t_{(46)}=2.53 ; p=0.015 ; R^{2}=0.12\right)$. A series of four ANOVAs over $2 \mathrm{~ms}$ spans beginning $2 \mathrm{~ms}$ before P1 then continuing out to the 0 to $2 \mathrm{~ms}$ band, the 2 to $4 \mathrm{~ms}$ band, and the 4 to $6 \mathrm{~ms}$ band after P1 each provided significance (all $p<0.05$ ) for interactions between latency trends and genotype, indicating that the amplitude difference between the groups was not constant across the waveform. Figure $2 B$ includes the latency regions in which the difference between the groups exceeded the $95 \%$ confidence interval, which tend to coincide with the end of a trough and the beginning rise to the next peak.

Figure $2 C$ depicts the mean (SEM) latency differences between $+/+$ and $-/-$ mice for each peak and trough feature of the waveform. There was no difference in raw P1 latencies: $+/+$ mice, $2.250 \mathrm{~ms}(0.024 \mathrm{~ms}) ;-/-$ mice, $2.255 \mathrm{~ms}(0.017 \mathrm{~ms})$. The difference between groups showing more rapid transmission in the $-1-$ group was small but significant: for the first negative peak, N1, $t_{(46)}=3.10, p=0.003$, and $R^{2}=0.17$; for $\mathrm{P} 2, t_{(46)}=$ $3.30, p=0.002$, and $R^{2}=0.19$; for $\mathrm{N} 3, t_{(46)}=2.33, p=0.024$, and $R^{2}=0.11$; and for P4, $t_{(46)}=2.941, p=0.005$, and $R^{2}=0.16$. The ANOVA of the data in Figure $2 C$ provided a highly significant main effect of waveform feature $\left(F_{(6,276)}=3369.89 ; p<\right.$ $\left.0.001 ; \eta_{p}^{2}=0.99\right)$ and for the interaction of genotype and the linear trend for features $\left(F_{(1,46)}=5.00 ; p=0.03 ; \eta_{p}{ }^{2}=0.10\right)$. In 

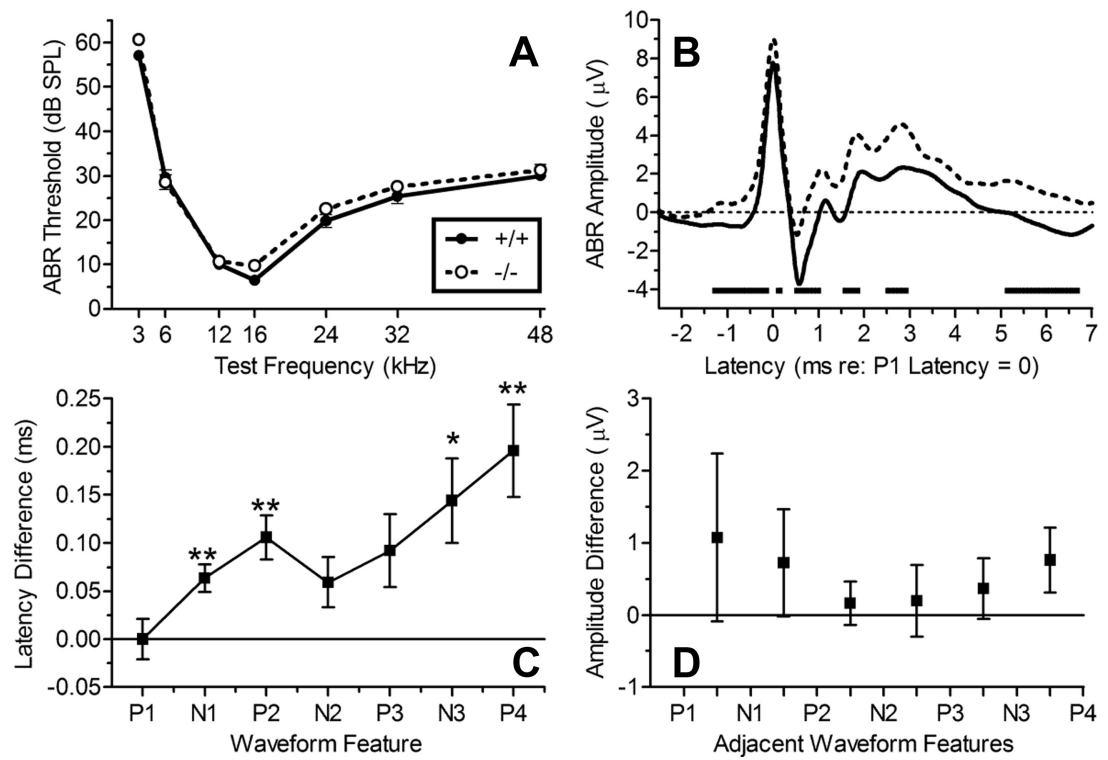

Figure 2. Normal ABR thresholds and abnormal suprathreshold waveforms in Kcna1-/- and Kcna1+/+ mice $(n=24,24)$. $A$, Mean (SEM) thresholds for each test frequency, showing the significant $(p=0.001) \mathrm{U}$-shaped function typical of mice with their best frequency at $16 \mathrm{kHz}$. The thresholds did not significantly differ between the two groups $(p>0.1)$. $\boldsymbol{B}$, The mean waveform in each group for the $16 \mathrm{kHz}, 80 \mathrm{~dB}$ SPL test stimulus. There was an overall greater amplitude in the $-/-$ mice $(p=0.02)$. The broken horizontal line at -4 on the ordinate marks the times at which the two waveforms were significantly different $(p<0.05)$, typically coinciding with the earlier beginning rise from a trough toward the next peak in the $-/-$ mice. $C$, The mean (SEM) latency differences between $+/+$ and $-/-$ mice of the sequence of ABR peaks and troughs. Latencies were faster for $-/-$ mice $(p<0.001)$, and the difference increased with increasing feature number $(p=0.03)$. $\boldsymbol{D}$, The mean (SEM) amplitude difference in peak-to-trough potentials showed no effect of genotype $(p=0.6) .{ }^{*} p<0.05 ;{ }^{* *} p<0.01$.
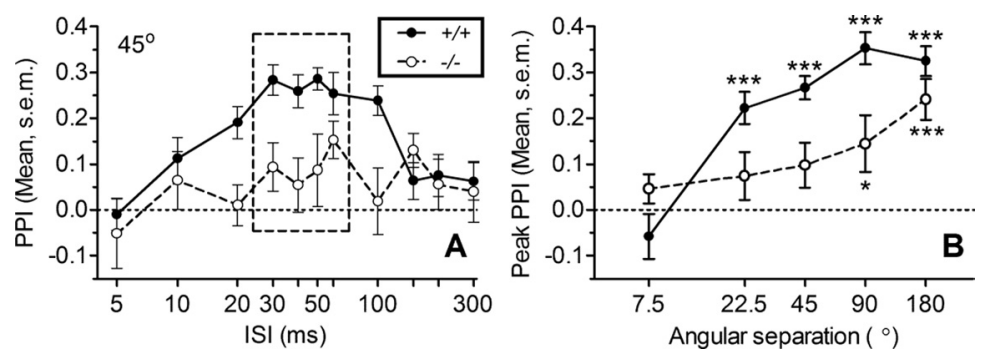

Figure 3. Impaired spatial acuity in Kcna1-null mutant mice. $\boldsymbol{A}$, Mean (SEM) PPI produced by the $45^{\circ}$ change in sound location for Kcna1-/- $(N=10)$ and $+/+$ control mice $(N=13)$ for different time intervals (ISIs) between the change in speaker and delivery of the startle stimulus. ANOVA indicate that the peak PPI response (dotted box) of the inverse-U-shaped curve differed by genotype $(p=0.005)$. $B$, Mean (SEM) peak PPI response versus angle. The response was strong in $+/+$ control mice down to $22.5^{\circ}$, while for Kcna1 - / - mice it was weak below $180^{\circ}$. Post hoc testing used the Wilcoxon signed rank test for peak PPI responses significantly greater than zero. ${ }^{*} p<0.05 ;{ }^{* * *} p<0.001$.

short, the difference in ABR latencies between the genotypes is highly significant and increases with increasing ABR feature number, indicating a cumulative process, leading to an increased disparity between the groups for later peaks and troughs.

Figure $2 D$ shows mean (SEM) amplitude difference in peak-totrough potentials between neighboring features, beginning with the (negative) change in amplitude from $\mathrm{P} 1$ to $\mathrm{N} 1$ and ending with the (positive) change in amplitude from $\mathrm{N} 3$ to $\mathrm{P} 4$. Here, despite the significant overall higher level of neural activity in the $-/-$ mice, there are no differences apparent in the overall amplitude of the excursions between one feature and the next, the ANOVA providing $F_{(1,46)}=0.25$, and the interaction between genotype across features yielding $F_{(5,230)}=1.21$ and $p>0.3$. The mean difference overall between the two groups was $0.174 \mu \mathrm{V}$, and the $95 \%$ confidence interval extended from +0.861 to $-0.516 \mu \mathrm{V}$.
Impaired behavioral auditory spatial acuity in mice lacking Kv1.1

Auditory spatial acuity was impaired in Kcna1-/- mice, as illustrated in Figure $3 A$ in the reduced PPI effect of a $45^{\circ}$ location shift compared with $+/+$ controls. There was an inverted-U-shaped trend in PPI for both groups as the ISI between moving the noise from one position through a $45^{\circ}$ angle to a second position and the ISI for the startle probe was varied from 5 to $300 \mathrm{~ms}$ (the 1 and $2 \mathrm{~ms}$ points equaled the control levels and for clarity are not included in this figure). The behavioral effect of this shift in location was lower in the null mutant mice: for the quadratic trend, $F_{(1,18)}=44.3, p=0.001$, and $\eta_{p}^{2}=0.71$. This inverted-U pattern with a peak level of inhibition centered at $\sim 50 \mathrm{~ms}$ was replicated at each angle save for $7.5^{\circ}$.

Figure $3 B$ depicts how the peak PPI (means of 40, 50, and $60 \mathrm{~ms}$ ISI) varied by angle for the two groups and shows that, compared to the $+/+$ group, performance in the $-/-$ group more rapidly declined as the angular separation was reduced below $180^{\circ}$. The ANOVA of peak performance provided significant effects for genotype $\left(F_{(1,85)}=14.22 ; p<0.001 ; \eta_{p}^{2}=0.43\right)$, angular separation $\left(F_{(4,85)}=13.67 ; p<0.001\right.$; $\eta_{p}^{2}=0.31$ ), and the interaction of genotype and angular separation $\left(F_{(4,85)}=3.96 ; p=\right.$ $\left.0.005 ; \eta_{p}^{2}=0.15\right)$. For the $180^{\circ}$ separation, the groups were not significantly different from each other and both provided significant levels of PPI, but the group differences were significant for the 90,45 , and $22.5^{\circ}$ separations. Also, for these three angles, the average behavioral effect for $+/+$ mice was always significantly above zero $(p<0.05)$, while for the $-/-$ mice it was never significant, and at $7.5^{\circ}$ neither group had a significant PPI above zero. The major contribution of the $7.5^{\circ}$ data is to show that any spectral difference that might be present between the two speakers was not sufficient to have a behavioral effect.

Smart et al. (1998) remarked that Kcna1-/- mice have an exaggerated ASR but did not provide any supporting evidence for their observation. In the current experiment, the $+/+$ mice responded more vigorously, with a mean response (SD) of $1622 \mathrm{aV}$ (533 aV) compared to $893 \mathrm{aV}(271 \mathrm{aV})$ for the $-/-$ mice: $t_{(21)}=3.93, p=$ 0.001 , and $R^{2}=0.42$. Less vigorous responding of Kcna1 $-/-$ mice compared to $+/+$ mice was reported also by $\operatorname{Karcz}(2011)$, especially for normal listening conditions, the difference between the groups being reduced when one ear was plugged.

\section{Discussion}

Behavioral responses to changes in sound location

The hypothesis that the Kcnal gene contributes to the ability of mice to localize sounds has its foundation in the in vitro and in vivo experiments reporting that the principal monaural and bin- 
aural neurons in the brainstem pathways that transmit and encode IID stimuli lose their high level of temporal synchrony between neural inputs and response outputs in Kcna1-/- mice (Brew et al., 2003; Kopp-Scheinpflug et al., 2003; Gittelman and Tempel, 2006; Karcz et al., 2011). This hypothesis is further supported by recent in vivo data showing that at the level of the midbrain IC, the Kcna1-/- mice are sensitive to temporally displaced inhibitory inputs that lie outside the normal temporal window for coincidence integration (Karcz, 2011). The behavioral experiment reported here also supports this hypothesis by showing that $-/-$ mice were less able to detect changes in the location of a noise background over angular separations from the near-localization threshold for mice at $22.5^{\circ}$ (Heffner and Heffner, 1988; Allen and Ison, 2010) and up to 45 and $90^{\circ}$. Two control measures anchored these results: at the widest separation of $180^{\circ}$, both groups detected these stimuli, and at the lowest separation of $7.5^{\circ}$, neither group detected them. Based on the study by Chen et al. (1995), who reported interaural attenuations in the mouse, we estimate that an IID of $\sim 35 \mathrm{~dB}\left(180^{\circ}\right)$ was an effective stimulus in both $\mathrm{Kcna1}+/+$ and Kcna1 $-/-$ mice, while an IID of $5 \mathrm{~dB}\left(22.5^{\circ}\right)$ was effective for the $+/+$ mice, but not for the $-/-$ mice.

\section{Implications of the $\mathrm{ABR}$ data for understanding the behavioral results}

One apparent difference in outcome between the in vitro experiments that stimulate Kcna1-/- cells with current injection (Brew et al., 2003; Gittelman and Tempel, 2006) and the in vivo experiments that use sound stimulation (Kopp-Scheinpflug et al., 2003; Karcz et al., 2011; Karcz, 2011) is the in vitro finding of lower response thresholds and hyperreactivity in the $-/-$ cells, contrasted with the in vivo finding of higher response thresholds and hyporeactivity in $-/-$ mice. One hypothesis that would explain these differences in response thresholds is that the Kcna1-/- mouse has a peripheral hearing deficit that affects their responses to tones but not to current injection, this being plausible given the normal high expression of this gene in the spiral ganglion cells of the auditory nerve (Adamson et al., 2002). Furthermore, hearing loss might increase the temporal dispersion of onset responses to suprathreshold tonal stimuli as found in the in vivo experiments, and also reduce their performance in the behavioral experiment, as this too depends in part on stimulus intensity (Allen and Ison, 2010). The evoked potential experiment in anesthetized mice was designed to test this hypothesis in anesthetized mice using the ABR test to compare the hearing thresholds of $+/+$ and $-/-$ mice: as shown in Figure $2 A$, their thresholds from $3 \mathrm{kHz}$ up to $48 \mathrm{kHz}$ were close to identical. Alternative hypotheses to explain the difference between the in vitro and in vivo observations that would apply to behavioral experiments as well have been suggested (Kopp-Scheinpflug et al., 2003): cells studied in vivo are affected by the possibly enhanced inhibitory inputs from other different cell types, and their excitability is also dependent on more peripheral axonal input, which can be blocked by the greater occurrence of backfiring and collision in -/- cells (Chui et al., 1999).

The suprathreshold ABR waveform may help explain other effects of Kv1.1 deletion. The group average ABR waveforms depicted in Figure $2 B$ mark the passage of peaks of synchronous depolarization and troughs of synchronous repolarization and recovery of neural generators. These have been described for the mouse (Henry, 1979) and include the afferent pathway important for sound localization: the cochlear nucleus, the superior olivary complex, and the nuclei and pathways of the lateral lem- niscus that then access the midbrain nuclear groups of the inferior colliculus. Evident in Figure $2 B$ is the presence of a significant level of stimulus-evoked but nonsynchronized activity in the $-/-$ mice that might be the evoked potential counterpart of the nonsynchronized hyperactivity typical of in vitro observations. Other than this shift in firing level, the topography was very similar for the $+/+$ and $-/-$ waveforms (Fig. $2 D$ ) save for the slightly more rapid transmission of synchronous activity beginning with the earlier return of depolarization (N1) that followed auditory nerve firing in $-/-$ mice and increasing on up through the brainstem. This is a classic example of the effect of blocking Kv1.1 subunits, because in the absence of this most sensitive mechanism for repolarizing the cell membrane, the next round of depolarization culminating in the next peak must begin more quickly. We note, however, that the maximal latency difference between the two groups is just $0.2 \mathrm{~ms}$, and while this may be an interesting symptom of neural dysfunction, it seems doubtful that by itself it would substantially disrupt normal auditory processing; indeed auditory temporal processing at the severalmillisecond time scale is intact in these mice as measured via gap detection (Allen et al., 2008). The likely more serious consequence of Kcnal deletion seen in the ABR waveform is its allowing significant levels of asynchronous and "noisy" neural activity to occur in the brainstem, which could present a more serious challenge to the relatively normal processing of IID signals reflected in the similar amplitudes of the excursions from one waveform feature to the next.

\section{Conclusions: the several auditory and nonauditory brainstem sites that contribute to sound localization that may be affected by Kcnal deletion}

The hypothesis that Kcnal deletion in the mouse would reduce its sensitivity to changes in the location of a sound source was confirmed in the behavioral experiment, and the similar hearing thresholds obtained in the ABR experiment indicate that Kcnal had a direct effect on the behavior, rather than an indirect effect resulting from a peripheral hearing loss. The specific locus of the dysfunctional central neural sites affected by Kcnal deletion cannot be determined at present, because in addition to the particular chain of monaural and binaural nuclei investigated in the electrophysiological experiments on which this hypothesis was based, there are other nuclei and other pathways where Kcnal expression is found and that contribute to sound localization.

The apparent spectral composition of complex stimuli varies depending on the relative positions of the sound and the listener because of the directional filtering properties of the head and ears [for humans, see the study by Shaw (1974); for cats, see the study by Rice et al. (1992)], and this has been shown to provide a significant cue for monaural sound localization [in humans (Butler, 1986) and in cats (May, 2000)]. Karcz (2011) found that monauralized mice had reduced but still significant inhibition for shifts in azimuthal location over 90 and $120^{\circ}$. This monaural effect was also diminished by Kcnal deletion. Electrophysiological correlates of spectral filtering have been found in the dorsal cochlear nucleus (DCN) (Spirou and Young, 1991), and Kv1.1 is known to be expressed in the DCN (Grigg et al., 2000) and can be expected to contribute to its processing complex spectral stimuli.

Both monaural spectral and binaural interaural IID and ITD cues to sound localization must fluctuate with bodily movements and, for the mouse, with changes in the orientation of the pinna, as they do for the mobile pinna of the cat (Young et al., 1996). Accurate sound localization may require stabilization of acoustic space, e.g., via a vestibuloauricular reflex (Tollin et al., 2009) in 
the same way that the vestibulo-ocular reflex stabilizes visual space (Lorente de No 1933). Movement-generated fluctuations of the sound field must be distinguished from external changes in the position of sound sources, and this may be the province of the vestibular inputs expressing Kv1.1 (Kalluri et al., 2010) and similar somatosensory and proprioceptive inputs into the brainstem auditory nuclei (Shore and Zhou, 2006). These multisensory cells have been most actively investigated in the DCN (Kanold et al., 2011), and while their functional significance is not fully understood, it is thought that DCN circuits and others, in the IC perhaps, must play a modulatory role in sound localization (Oertel and Young, 2004). To the extent that these circuits depend on the temporal coincidence of, for example, fluctuations in IID and proprioceptive and movement cues, it may be that they would also be disrupted by Kcnal deletion. The investigation of these potential site-specific contributions of Kcnal will depend on the development of site-specific changes in Kcnal expression. Of particular of interest would be joint electrophysiological and behavioral investigations of these suggested cross-modal effects necessary for disambiguating external from internal influence sources on the proximal acoustic cues for sound localization.

\section{References}

Adamson CL, Reid MA, Mo Z-L, Bowne-English J, Davis RL (2002) Firing features and potassium channel content of murine spiral ganglion neurons vary with cochlear location. J Comp Neurol 447:331-350.

Allen PD, Ison JR (2010) Sensitivity of the mouse to changes in azimuthal sound location: effects of angular separation, spectral composition, and sound level on prepulse inhibition of the acoustic startle reflex. Behav Neurosci 124:265-277.

Allen PD, Schmuck N, Ison JR, Walton JP (2008) Kv1.1 channel sub-units are not necessary for high temporal acuity in behavioral and electrophysiological gap detection. Hear Res 246:52-58.

Brew HM, Hallows J, Tempel BL (2003) Hyperexcitability and reduced low threshold potassium currents in auditory neurons of mice lacking the channel subunit Kv1.1. J Physiol 548:1-20.

Butler RA (1986) The bandwidth effect on monaural and binaural localization. Hear Res 21:67-73.

Chen QC, Cain D, Jen P H-S (1995) Sound pressure transformation at the pinna of mus domesticus. J Exp Biol 198:2007-2023.

Cherry EC (1953) Some experiments of the recognition of speech, with one and with two ears. J Acoust Soc Am 25:975-979.

Chui SY, Zhou L, Zhang CL, Messing A (1999) Analysis of potassium channel functions in mammalian axons by gene knockouts. J Neurocytol 28:349-364.

Feng AS, Ratnam R (2000) Neural basis of hearing in real-world situations. Annu Rev Psychol 51:699-725.

Gittelman JX, Tempel BL (2006) Kv1.1-containing channels are critical for temporal precision during spike initiation. J Neurophysiol 96:1203-1214.

Grigg JJ, Brew HM, Tempel BL (2000) Differential expression of voltagegated potassium channel genes in auditory nuclei of the mouse brainstem. Hear Res 140:77-80.

Heffner RS, Heffner HE (1988) Sound localization in a predatory rodent, the northern grasshopper mouse (Onychomys leucogaster). J Comp Psychol 102:66-71.
Henry KR (1979) Auditory brainstem volume-conducted responses: origins in the laboratory mouse. J Acoust Soc Am 4:173-178.

Hershkowitz RM, Durlach NI (1969) Interaural time and amplitude jnds for a 500-Hs tone. J Acoust Soc Am 46:1464-1467.

Joris PX, Yin TC (1995) Envelope coding in the lateral superior olive. I. Sensitivity to interaural time differences. J Neurophysiol 73:1043-1062.

Kalluri R, Xue J, Eatock RA (2010) Ion channels set spike timing regularity of mammalian vestibular afferent neurons. Neurophysiol 104:2034-2051.

Kanold PO, Davis KA, Young ED (2011) Somatosensory context alters auditory responses in the cochlear nucleus. J Neurophysiol 105:1063-1070.

Karcz A (2011) The significance of the low voltage-gated potassium channel subunit Kv1.1 for the processing of sound source location. PhD Dissertation, University of Leipzig.

Karcz A, Hennig MH, Robbins CA, Tempel BL, Rubsamen R, KoppScheinpflug C (2011) Low-voltage activated Kv1.1 subunits are crucial for the processing of sound source location in the lateral superior olive in mice. J Physiol 589:1143-1157.

Kopp-Scheinpflug C, Fuchs K, Lippe WR, Tempel BL, Rubsamen R (2003) Decreased temporal precision of auditory signaling in Kcnal-null mice: An electrophysiological study in vivo. J Neurosci 23:9199-9207.

Lorente de No R (1933) The vestibulo-ocular reflex arc. Arch Neurol Psychiatry Chicago 33:245-291.

May BJ (2000) Role of the dorsal cochlear nucleus in the sound localization behavior of cats. Hear Res 148:74-87.

Mills AW (1958) On the minimum audible angle. J Acoust Soc Am 30:237-246.

Oertel D (1999) The role of timing in the brain stem auditory nuclei of vertebrates. Annu Rev Physiol 61:497-519.

Oertel D, Young ED (2004) What's a cerebellar circuit doing in the auditory system? Trends Neurosci 27:104-110.

Oertel D, Bal R, Gardner SM, Smith PH, Joris PX (2000) Detection of synchrony in the activity of auditory nerve fibers by octopus cells of the mammalian cochlear nucleus. Proc Natl Acad Sci U S A 97:11773-11779.

Rice JJ, May BJ, Spirou GA, Young ED (1992) Pinna-based spectral cues for sound localization in cat. Hear Res 58:132-152.

Rosenberger MH, Fremouw T, Casseday JH, Covey E (2003) Expression of the Kv1.1 ion channel subunit in the auditory brainstem of the big brown bat, Eptesicus fuscus. J Comp Neurol 462:101-120.

Shaw EA (1974) Transformation of sound pressure level from the free field to the eardrum in the horizontal plane. J Acoust Soc Am 56:1848-1861.

Shore SE, Zhou J (2006) Somatosensory influence on the cochlear nucleus and beyond. Hear Res 216-217:90-99.

Smart SL, Lopantsev V, Zhang CL, Robbins CA, Wang H, Chiu SY, Schwartzkroin PA, Messing A, Tempel BL (1998) Deletion of the K(V)1.1 potassium channel causes epilepsy in mice. Neuron 20:809-819.

Spirou GA, Young ED (1991) Organization of dorsal cochlear nucleus type IV unit response maps and their relationship to activation by bandlimited noise. J Neurophysiol 66:1750-1768.

Tollin DJ, Ruhland JL, Yin TC (2009) The vestibulo-auricular reflex. J Neurophysiol 101:1258-1266.

Trussell LO (1999) Synaptic mechanisms for coding timing in auditory neurons. Annu Rev Physiol 61:477-496.

Yin TC (2002) Neural mechanisms of encoding binaural localization cues in the auditory brainstem. In: Integrative functions in the mammalian auditory pathway (Oertel D, Fay RR, Popper AN, eds), pp 99-159. New York: Springer.

Young ED, Rice JJ, Tong SC (1996) Effects of pinna position on headrelated transfer functions in the cat. J Acoust Soc Am 99:3064-3076. 